\title{
Intramuscular Neural Distribution of the Serratus Anterior Muscle: Guidelines to the Injective Method for Treating Myofascial Pain Syndrome
}

Kyu-Ho Yi

Yonsei University

Ji-Hyun Lee

Yonsei University

Kyle K Seo

Modelo Clinic

Hee-Jin Kim ( $\nabla$ hjk776@yuhs.ac)

Yonsei University

\section{Research Article}

Keywords: myofascial pain syndrome, sihler's method, serratus anterior, trigger point injection

Posted Date: February 15th, 2021

DOI: https://doi.org/10.21203/rs.3.rs-190375/v1

License: (c) (i) This work is licensed under a Creative Commons Attribution 4.0 International License. Read Full License 


\section{Abstract}

The serratus anterior muscle is commonly involved in myofascial pain syndrome and is treated with many different injective methods. Currently, there is no definite injection point for the muscle. This study provides an ideal injection point for the serratus anterior muscle considering the intramuscular neural distribution using the whole mount staining method. A modified Sihler method was applied to the serratus anterior muscles (15 specimens). The intramuscular arborization areas were identified in terms of the anterior $(100 \%)$, middle (50\%), posterior axillary line $(0 \%)$, and from the first to the ninth ribs. The intramuscular neural distribution for the serratus anterior muscle had the largest arborization patterns in the 5 th to 9 th rib portion between $50 \%$ and $70 \%$, and the 1 st to 4 th rib portion had between $20 \%$ and $40 \%$. Clinicians can administer safe and effective treatments with botulinum neurotoxin injections and other types of injections, following the methods in our study. We propose optimal injection sites in relation to the external anatomical line for the frequently injected facial muscles to facilitate the efficiency of botulinum neurotoxin injections. Lastly, these guidelines would assist practice more accurately without the harmful side effects of trigger point injections and botulinum neurotoxin injections.

\section{Introduction}

Myofascial pain syndrome (MPS) is extremely common, occurring in up to $95 \%$ of individuals; 9 million cases have been diagnosed in the United States ${ }^{1}$. Repetitive movements and incorrect posturing habits contribute to the advancement of MPS by triggering overload on a particular muscle; the serratus anterior (SA) muscle is the most commonly involved ${ }^{2}$. As a part of MPS, serratus anterior myofascial pain syndrome (SAMPS) is separately named for its frequency ${ }^{2}$. Points with taut banded parts and pinched tenderness of the muscle belly are termed myofascial trigger points (MTrPs). SAMPS occurs with deep respiratory distress while running, repetitive coughing due to respiratory disease, lifting heavy loads, and other psychological stresses ${ }^{3}$.

The cause of SAMPS is hyperactivated SA muscle contractions ${ }^{4-6}$. Pathological findings indicate an increase in the release of acetylcholine by the neuromuscular junction under relaxing conditions. Elevated and prolonged acetylcholine release generates persistent depolarization of the muscle fiber, which causes sarcomere shortening and involuntary muscle contraction ${ }^{2}$. This point is anatomically known to be the thickest muscle belly, with the most intramuscular neural arborization ${ }^{7-11}$.

The therapeutic options for MPS include releasing MTrPs using injective agents such as botulinum neurotoxin (BoNT), lidocaine, steroids, normal saline, and combinations of agents. BoNT blocks neural transmission by stalling the release of acetylcholine at the neuromuscular junction and impedes muscle contraction ${ }^{12}$. In myofascial pain control, BoNT injection is renowned for offering better consequences than oral medications in terms of pain management and functional movement ${ }^{13-15}$. Therefore, BoNT is widely used as a treatment option for MPS, especially SAMPS ${ }^{16-21}$. 
At present, BoNT injection is acknowledged as the most secure and effective treatment for inactivating the muscle ${ }^{22-25}$. The consequences of BoNT depend on uptake by the presynaptic membranes at the neuromuscular junction; thus, injections should be directed into the neuromuscular junction area where most neuromuscular junctions exist ${ }^{12,26,27}$. The significance of utilizing neuromuscular arborizationdirected BoNT injections has been verified by clinical trials in the iliopsoas and biceps brachii muscles. These injections resulted in higher pain reduction as well as volume reduction compared to conventional injections ${ }^{28,29}$.

However, a precise infusion point is necessary for BoNT, as excessive amounts of BoNT may potentially cause the toxin to spread to the neighboring muscles, resulting in paralysis ${ }^{30,31}$. The adverse effect of paralyzed muscle is reported in cases of overdose of BoNT ${ }^{32-34}$. Moreover, repetitive and overdose BoNT injections build up antibodies that will result in an insufficient treatment effect $30,31,35,36$. Additionally, a previous study indicated neuropathy due to mechanical damage subsequent to injection in the intramuscular nerve trunks and nerve entering point, where the nerve penetrates the muscle ${ }^{37}$. Consequently, BoNT should be injected into the arborized regions to enhance efficacy and decrease adverse effects. To direct the injection points while preventing these adverse effects, numerous studies have revealed the intramuscular neural arborization of various muscles, but not the SA ${ }^{14,38-41}$.

This study aimed to suggest efficient and secure injection points for SAMPS considering intramuscular neural arborization.

\section{Results}

\section{Running of the thoracic nerve trunk}

The long thoracic nerve runs superficial to the SA muscle and pierces the muscle at each level until the 7th rib. Thirteen of the 15 specimens had a trunk of the long thoracic nerve running on 30 to $50 \%$ throughout the level of the $1 \mathrm{st}$ to $7 \mathrm{th}$ rib. The other two had the long thoracic nerve running down on the 40 to $50 \%$ at the level of the 1 st to 4 th rib and 30 to $40 \%$ at the level of the 5 th to 7 th rib.

\section{Intramuscular arborization patterns of the SA muscle}

Twelve of the 15 SA muscles had two regions in which the arborization patterns were the largest: in the 6th to 9 th rib portion had between $50 \%$ and $70 \%$ and the 1 st to 5 th rib portion had between 20 and $40 \%$, following three anatomical lines: anterior (100\%), middle (50\%), and posterior axillary line (0\%) (Fig. 1). The other two had the largest patterns in the 4th to 9th rib portion, between $50 \%$ and $60 \%$; the 1 st to $3 \mathrm{rd}$ rib portion had between $20 \%$ and $30 \%$. The last muscle had the largest patterns in the 4 th to 9 th rib portion, between $50 \%$ and $70 \%$; the 1 st to 3 rd rib portion had between 30 to $40 \%$.

\section{Discussion}


The SA muscle is a flat and wide muscle covering the lateral ribs; it is anatomically divided into three muscle bellies ${ }^{2}$. It consists of an upper, middle, and lower muscle belly, each of which contribute to the movement of the scapular bone during upper extremity actions ${ }^{42}$. The upper belly of the SA lies parallel to the $1 \mathrm{st} \mathrm{rib}$ and inserts into the superior angle of the scapula ${ }^{43}$. The middle belly of the SA originates from the 2nd, 3rd, and 4th ribs and inserts into the medial scapular border ${ }^{43}$. The lower muscle belly of the SA is where the MTrPs frequently exist, originating from the 5th to the 9th ribs and inserting into the inferior angle of the scapula 43,44 . The SA muscle is innervated by the long thoracic nerve, which originates from the anterior rami of spinal nerves $\mathrm{C} 5-\mathrm{C} 7{ }^{45}$. The long thoracic nerve runs superficially over the SA muscle along the anterior axillary line. The SA muscle is mostly involved in upper extremity movements; however, it is the prime stabilizer of the shoulder girdle and acts on shoulder flexion, abduction, and upward rotation ${ }^{42}$.

MPS is a chronic pain disorder caused by MTrPs situated at the muscle belly; it has been recognized as the main cause of pain in $85 \%$ of patients attending pain clinics ${ }^{46,47}$. SA muscle MTrPs may be triggered by muscle strain during excessive running, overloaded weight lifting, or repetitive coughing, especially susceptible to torsional stresses. Another cause of MTrPs initiation in the SA muscle is breast surgery due to cancer or esthetic purposes ${ }^{48}$.

Studies have revealed that sarcomere shortening is related to MTrPs etiology, and the shortening is due to an increase in activation of the neuromuscular junction and its over-release of acetylcholine. In addition, a large quantity of calcium released at the sarcoplasmic reticulum over a dysfunctional ryanidine receptor causes prolonged muscle contraction ${ }^{49}$. Therefore, to release muscle contraction, BoNT is currently frequently used as an injective agent for MPS ${ }^{49-51}$. The primary known therapeutic effects are by releasing muscular contractions and alleviating the vicious pain cycle ${ }^{52-54}$. It is also thought that the relief from the muscle tightness and the BoNT inhibit the diffusion of neurotransmitters in the peripheral nerve, avoiding peripheral sensitization ${ }^{55,56}$.

In treating MPS, it is critical to locate MTrPs, and electromyography (EMG) may help to verify the presence of MTrPs, as they are thought to have sensitized nerves that spontaneously produce lowamplitude electrical activity 7,57-59. The study by Kuan et al. demonstrated BoNT injection in MTrPs and found that the injection diminished spontaneous electrical activity in MTrPs ${ }^{60}$. There are many studies indicating that MTrP pathophysiology appears to be associated with the intramuscular neural arborized areas, also known as identical with MTrPs ${ }^{7-11}$. A study by Xie et al. showed that both BoNT injection and lidocaine injection therapy in the intramuscular arborized area substantially lessens the intensity and frequency of pain in patients ${ }^{18}$.

Vargas-Schaffer et al. ${ }^{61}$ performed trigger point injection in patients with SAMPS and showed its effectiveness in pain control after three months of injective treatment. In 8 patients, the duration of SAMPS was approximately 19 months. With the patient in a lateral decubitus position, the injections were conducted over the midaxillary line on the 5th to the 7 th ribs. 
Many studies have described frequent SAMPS incidence after breast cancer treatment ${ }^{48,62-64}$. Lacomba et al. ${ }^{48}$ reported a prevalence rate of $45 \%$ for SAMPS one year after breast cancer surgery. Layeeque et al. 21 observed 48 breast cancer patients who underwent mastectomy and observed post-surgical pain; 22 patients were treated with BoNT in the SA and pectoralis major muscle, and 26 control group patients were treated with medication. The BoNT-treated group reported significant pain reduction compared to the pain medication group.

Neuromuscular junctions are the underlying causes of MPS; therefore, injecting BoNT and other injective treatments such as lidocaine, steroids, and normal saline are frequently performed to target the neuromuscular junctions ${ }^{65-67}$. Unlike oral medications and lidocaine injections that have short-term effects, the effectiveness of BoNT treatment in MPS has been known to continue for up to 4 months ${ }^{66,67}$.

As BoNT acts on the neuromuscular junction, accurate anatomical knowledge of the neuromuscular arborization patterns of the SA muscles is vital for achieving the highest relief with the smallest possible dose of BoNT. Although BoNT procedures are minimally invasive compared to surgical intervention, there is a probability of damaging the nerve trunks that are not present near the neural arborized area.

Therefore, precise knowledge of the anatomical features of the SA muscle should be considered. In this study, we carried out Sihler's staining, which is a whole mount staining procedure that stains myelin sheaths and is effective in tracing the nerve endings without destroying the nerves ${ }^{14,38-40,68}$. The application of Sihler's staining to the SA muscle will enable an accurate and thorough understanding of the neural distribution.

Moreover, identifying the neural arborization area of the SA muscle is important in diagnosing long thoracic nerve palsy ${ }^{69}$. Surface electromyography in the SA muscle is challenging because multiple thin digitations make it difficult to place the electrode for recording ${ }^{70}$. When detecting long thoracic nerve palsy, the technical limitations of electromyography are interrupted signals from the neighboring muscles and difficulty with accurate electrode placement since the SA is not a bulky muscle.

At present, there is no standardized injection or EMG points of the SA muscle. In this study, we suggest that electromyography and injective treatments including BoNT, lidocaine, normal saline, steroids should be administered in the three regions in the middle portion, between the 6th to 9th rib portion and the 1st to 5th rib (Fig. 2). Surgeons should be aware that they must inject over the bony rib with patients holding their breath to avoid iatrogenic pneumothorax.

\section{Methods}

This study was performed in accordance with the principles outlined in the Declaration of Helsinki. Informed consent and approval were obtained from the families of the cadavers before the dissections were performed. All cadavers used in this study were legally donated and approved from ethics committee of the Surgical Anatomy Education Center, Yonsei University College of Medicine (approval code 20 - 006; approval date: May 5th, 2020). A total of 15 SA muscles from Korean cadavers (5 men 
and 4 women with a mean age of 76.6 years; range, 73-95 years) were dissected and modified Sihler staining was applied to clarify the intramuscular neural arborization patterns.

Before dissection, the SA muscles were aligned in their anatomical positions. The arborizing patterns of the SA muscles were tracked according to the three anatomical lines: anterior (100\%), middle (50\%), posterior axillary line (0\%), and from the first to the ninth ribs (Fig. 3 ).

The SA muscles underwent Sihler staining, as modified by Liem and Douwe van Willingen ${ }^{71}$.

This technique involves several steps to acquire the visual representation of the intramuscular neural arborization pattern. The changes over Sihler's method of the SA specimens are shown in Fig. 4.

Following Sihler staining, the SA muscles were divided into 10 sections according to the vertical lines from the anterior and posterior axillary lines and the curved lines of the first to ninth ribs.

\section{Modified Sihler staining}

Fixation. The SA muscles were stored for one month in a container filled with $10 \%$ un-neutralized formalin. The solution was replaced with fresh solution whenever it turned cloudy.

Maceration and depigmentation: the fixed SA specimens were washed in running water for an hour. Then, they were placed for one month in a container filled with $3 \%$ aqueous potassium hydroxide and hydrogen peroxide solution.

Decalcification: the depigmented SA specimens were then placed in Sihler I solution, a compound of glycerin, glacial acetic acid, and aqueous chloral hydrate.

Staining: the decalcified SA specimens were then stained with the Sihler II solution, a compound of glycerin, aqueous chloral hydrate, and acetic acid. The staining process takes $30-35$ days for intramuscular nerve visualization.

De-staining: the stained SA specimens were cleansed in a container filled with Sihler I solution. This step is to de-stain the SA muscle fibers so that only the intramuscular nerve distributions are visualized.

Neutralization: the de-stained SA specimens were neutralized in clean water for half an hour. Consequently, the SA specimens were placed in a solution of $0.05 \%$ lithium carbonate.

Clearing: finally, the neutralized SA specimens were taken into the clearing stage with glycerin by increasing the concentrations from $20-100 \%$. This stage took nearly $4-5$ hours.

\section{List Of Abbreviations}


Myofascial pain syndrome (MPS), serratus anterior (SA), serratus anterior myofascial pain syndrome (SAMPS), myofascial trigger points (MTrPs), botulinum neurotoxin (BoNT), maceration and depigmentation (MD), decalcification, staining (ST), and clearing (CL), anterior axillary line (AA), posterior axillary line (PA)

\section{Declarations}

\section{Acknowledgments}

The authors sincerely thank those who donated their bodies to science so that anatomical research could be performed. Results from such research can potentially increase mankind's overall knowledge that can then improve patient care. Therefore, these donors and their families deserve our highest gratitude.The authors thank Eun-Byul Yi from Eonbuk elementary school for illustrations. This work was supported by the National Research Foundation of Korea (NRF) grant funded by the Korean government (MSIP) (NRF2020R1A2B5B0100223811 and NRF-2019R1C1C101077612). This work was also supported by the Korea Medical Device Development Fund grant funded by the Korea government (the Ministry of Science and ICT, the Ministry of Trade, Industry and Energy, the Ministry of Health \& Welfare, the Ministry of Food and Drug Safety) (Project Number: 202012D01).

\section{Author Contribution}

KHY: research concept, study design, and writing of the manuscript

JHL: research concept and study design

KKS: literature review and data collection

HJK: reviewing/editing a draft of the manuscript, Supervise the manuscript

\section{Funding}

Not applicable

\section{Conflicts of interest/Competing interests}

The author(s) declare no competing interests.

\section{References}

1. Alvarez, D. J. \& Rockwell, P. G. Trigger points: diagnosis and management. Am Fam Physician. 65, 653-660 (2002). 
2. Bautista, A., Webb, C. \& Rosenquist, R. Serratus Anterior Muscle Pain Syndrome: A Diagnostic Conundrum. Pain Med. 18, 1600-1602 https://doi.org/10.1093/pm/pnw350 (2017).

3. Donnelly, J. M., Simons, D. G. \& Travell Simons \& Simons' myofascial pain and dysfunction: the trigger point manual. (2019).

4. Lee, S. T. et al. Changes in Activation of Serratus Anterior, Trapezius and Latissimus Dorsi With Slouched Posture. Ann Rehabil Med. 40, 318-325 https://doi.org/10.5535/arm.2016.40.2.318 (2016).

5. Manfredini, D., Cocilovo, F., Stellini, E., Favero, L. \& Guarda-Nardini, L. Surface electromyography findings in unilateral myofascial pain patients: comparison of painful vs. non painful sides. Pain Med. 14, 1848-1853 https://doi.org/10.1111/pme.12159 (2013).

6. Szyszka-Sommerfeld, L., Machoy, M., Lipski, M. \& Wozniak, K. The Diagnostic Value of Electromyography in Identifying Patients With Pain-Related Temporomandibular Disorders. Front Neurol. 10, 180 https://doi.org/10.3389/fneur.2019.00180 (2019).

7. Saxena, A., Chansoria, M., Tomar, G. \& Kumar, A. Myofascial pain syndrome: an overview. J Pain Palliat Care Pharmacother. 29, 16-21 https://doi.org/10.3109/15360288.2014.997853 (2015).

8. Lluch, E. et al. Effects of deep cervical flexor training on pressure pain thresholds over myofascial trigger points in patients with chronic neck pain. J Manipulative Physiol Ther. 36, 604-611 (2013).

9. Javanshir, K., Ortega-Santiago, R., Mohseni-Bandpei, M. A. \& Miangolarra-Page, J. C. \& Fernandez-deLas-Penas, C. Exploration of somatosensory impairments in subjects with mechanical idiopathic neck pain: a preliminary study. J Manipulative Physiol Ther. 33, 493-499 https://doi.org/10.1016/j.jmpt.2010.08.022 (2010).

10. Iglesias-Gonzalez, J. J., Munoz-Garcia, M. T., Rodrigues-de-Souza, D. P., Alburquerque-Sendin, F. \& Fernandez-de-Las-Penas, C. Myofascial trigger points, pain, disability, and sleep quality in patients with chronic nonspecific low back pain. Pain Med. 14, 1964-1970 https://doi.org/10.1111/pme.12224 (2013).

11. Duyur Cakit, B., Genc, H., Altuntas, V. \& Erdem, H. R. Disability and related factors in patients with chronic cervical myofascial pain. Clin Rheumatol. 28, 647-654 https://doi.org/10.1007/s10067-0091116-0 (2009).

12. Childers, M. K. Targeting the neuromuscular junction in skeletal muscles. Am J Phys Med Rehabil. 83, S38-44 https://doi.org/10.1097/01.phm.0000141129.23219.42 (2004).

13. Comella, C. L. The treatment of cervical dystonia with botulinum toxins. Journal of neural transmission. 115, 579-583 https://doi.org/10.1007/s00702-007-0831-4 (2008).

14. Yi, K. H. et al. Effective botulinum toxin injection guide for treatment of cervical dystonia. Clin Anat. 33, 192-198 https://doi.org/10.1002/ca.23430 (2020).

15. Vasileiadis, G. I., Sakellariou, V. I., Papagelopoulos, P. J. \& Zoubos, A. B. Posttraumatic focal dystonia of the shoulder. Orthopedics. 35, e977-980 https://doi.org/10.3928/01477447-20120525-47 (2012).

16. Climent, J. M., Kuan, T. S., Fenollosa, P. \& Martin-Del-Rosario, F. Botulinum toxin for the treatment of myofascial pain syndromes involving the neck and back: a review from a clinical perspective. Evid 
Based Complement Alternat Med 2013, 381459, doi:10.1155/2013/381459 (2013).

17. Kwanchuay, P. et al. Efficacy and Safety of Single Botulinum Toxin Type A (Botox(R)) Injection for Relief of Upper Trapezius Myofascial Trigger Point: A Randomized, Double-Blind, Placebo-Controlled Study. J Med Assoc Thai. 98, 1231-1236 (2015).

18. Xie, P. et al. Lidocaine Injection in the Intramuscular Innervation Zone Can Effectively Treat Chronic Neck Pain Caused by MTrPs in the Trapezius Muscle. Pain Physician. 18, E815-826 (2015).

19. Mor, N., Tang, C. \& Blitzer, A. Temporomandibular Myofacial Pain Treated with Botulinum Toxin Injection. Toxins (Basel). 7, 2791-2800 https://doi.org/10.3390/toxins7082791 (2015).

20. Chaurand, J., Pacheco-Ruiz, L., Orozco-Saldivar, H. \& Lopez-Valdes, J. Efficacy of botulinum toxin therapy in treatment of myofascial pain. J Oral Sci. 59, 351-356 https://doi.org/10.2334/josnusd.16-0614 (2017).

21. Layeeque, R. et al. Botulinum toxin infiltration for pain control after mastectomy and expander reconstruction. Ann Surg. 240, 608-613 discussion 613 - 604 https://doi.org/10.1097/01.sla.0000141156.56314.1f (2004).

22. Rosales, R. L. et al. Botulinum toxin injection for hypertonicity of the upper extremity within 12 weeks after stroke: a randomized controlled trial. Neurorehabil Neural Repair. 26, 812-821 https://doi.org/10.1177/1545968311430824 (2012).

23. Bhakta, B. B., Cozens, J. A., Bamford, J. M. \& Chamberlain, M. A. Use of botulinum toxin in stroke patients with severe upper limb spasticity. J Neurol Neurosurg Psychiatry. 61, 30-35 https://doi.org/10.1136/jnnp.61.1.30 (1996).

24. Brashear, A. et al. Intramuscular injection of botulinum toxin for the treatment of wrist and finger spasticity after a stroke. N Eng/ J Med. 347, 395-400 https://doi.org/10.1056/NEJMoa011892 (2002).

25. Hesse, S., Jahnke, M. T., Luecke, D. \& Mauritz, K. H. Short-term electrical stimulation enhances the effectiveness of Botulinum toxin in the treatment of lower limb spasticity in hemiparetic patients. Neurosci Lett. 201, 37-40 https://doi.org/10.1016/0304-3940(94)12124-9 (1995).

26. Ramirez-Castaneda, J. et al. Diffusion, spread, and migration of botulinum toxin. Mov Disord. 28, 1775-1783 https://doi.org/10.1002/mds.25582 (2013).

27. Childers, M. K. et al. Dose-dependent response to intramuscular botulinum toxin type A for upper-limb spasticity in patients after a stroke. Arch Phys Med Rehabil. 85, 1063-1069 https://doi.org/10.1016/j.apmr.2003.10.015 (2004).

28. Van Campenhout, A., Verhaegen, A., Pans, S. \& Molenaers, G. Botulinum toxin type A injections in the psoas muscle of children with cerebral palsy: muscle atrophy after motor end plate-targeted injections. Res Dev Disabil. 34, 1052-1058 https://doi.org/10.1016/j.ridd.2012.11.016 (2013).

29. Gracies, J. M. et al. Botulinum toxin dilution and endplate targeting in spasticity: a double-blind controlled study. Arch Phys Med Rehabil 90, 9-16 e12, doi:10.1016/j.apmr.2008.04.030 (2009).

30. Kinnett, D. Botulinum toxin A injections in children: technique and dosing issues. Am J Phys Med Rehabil. 83, S59-64 https://doi.org/10.1097/01.phm.0000141131.66648.e9 (2004). 
31. Hsu, T. S., Dover, J. S. \& Arndt, K. A. Effect of volume and concentration on the diffusion of botulinum exotoxin A. Arch Dermatol. 140, 1351-1354 https://doi.org/10.1001/archderm.140.11.1351 (2004).

32. Peng, H. P. \& Peng, J. H. Complications of botulinum toxin injection for masseter hypertrophy: Incidence rate from 2036 treatments and summary of causes and preventions. J Cosmet Dermatol. 17, 33-38 https://doi.org/10.1111/jocd.12473 (2018).

33. Song, J. H., Cho, E. S., Kim, S. T. \& Ahn, H. J. Change of distribution and timing of bite force after botulinum toxin type A injection evaluated by a computerized occlusion analysis system. Yonsei Med J. 55, 1123-1129 https://doi.org/10.3349/ymj.2014.55.4.1123 (2014).

34. Rafferty, K. L. et al. Botulinum toxin in masticatory muscles: short- and long-term effects on muscle, bone, and craniofacial function in adult rabbits. Bone. 50, 651-662 https://doi.org/10.1016/j.bone.2011.11.015 (2012).

35. Lepage, D., Parratte, B., Tatu, L., Vuiller, F. \& Monnier, G. Extra- and intramuscular nerve supply of the muscles of the anterior antebrachial compartment: applications for selective neurotomy and for botulinum toxin injection. Surg Radiol Anat. 27, 420-430 https://doi.org/10.1007/s00276-005-00129 (2005).

36. Pingel, J. et al. Injection of high dose botulinum-toxin A leads to impaired skeletal muscle function and damage of the fibrilar and non-fibrilar structures. Sci. Rep. 7, 14746 https://doi.org/10.1038/s41598-017-14997-3 (2017).

37. Lee, D. G. \& Chang, M. C. Dorsal scapular nerve injury after trigger point injection into the rhomboid major muscle: A case report. J Back Musculoskelet Rehabil. 31, 211-214 https://doi.org/10.3233/BMR-169740 (2018).

38. Yi, K. H. et al. Neuromuscular structure of the tibialis anterior muscle for functional electrical stimulation. Surg Radiol Anat. 39, 77-83 https://doi.org/10.1007/s00276-016-1698-6 (2017).

39. Yi, K. H. et al. Intramuscular nerve distribution pattern of ankle invertor muscles in human cadaver using sihler stain. Muscle Nerve. 53, 742-747 https://doi.org/10.1002/mus.24939 (2016).

40. Rha, D. W., Yi, K. H., Park, E. S., Park, C. \& Kim, H. J. Intramuscular nerve distribution of the hamstring muscles: Application to treating spasticity. Clin Anat. 29, 746-751 https://doi.org/10.1002/ca.22735 (2016).

41. Yi, K. H. et al. Guidelines for botulinum neurotoxin injections in piriformis syndrome. Clin Anat. https://doi.org/10.1002/ca.23711 (2020).

42. Hamada, J., Igarashi, E., Akita, K. \& Mochizuki, T. A cadaveric study of the serratus anterior muscle and the long thoracic nerve. J Shoulder Elbow Surg. 17, 790-794 https://doi.org/10.1016/j.jse.2008.02.009 (2008).

43. Moore, K. L., Agur, A. M. R. \& Dalley, A. F. Clinically oriented anatomy. (2019).

44. Webb, A. L., O'Sullivan, E., Stokes, M. \& Mottram, S. A novel cadaveric study of the morphometry of the serratus anterior muscle: one part, two parts, three parts, four? Anat Sci Int. 93, 98-107 https://doi.org/10.1007/s12565-016-0379-1 (2018). 
45. Nasu, H., Yamaguchi, K., Nimura, A. \& Akita, K. An anatomic study of structure and innervation of the serratus anterior muscle. Surg Radiol Anat. 34, 921-928 https://doi.org/10.1007/s00276-012-0984-1 (2012).

46. Fishbain, D. A., Goldberg, M., Meagher, B. R., Steele, R. \& Rosomoff, H. Male and female chronic pain patients categorized by DSM-III psychiatric diagnostic criteria. Pain. 26, 181-197 https://doi.org/10.1016/0304-3959(86)90074-6 (1986).

47. Tough, E. A., White, A. R., Richards, S. \& Campbell, J. Variability of criteria used to diagnose myofascial trigger point pain syndrome-evidence from a review of the literature. Clin J Pain. 23, 278-286 https://doi.org/10.1097/AJP.0b013e31802fda7c (2007).

48. Torres Lacomba, M., Mayoral del Moral, O., Coperias Zazo, J. L., Gerwin, R. D. \& Goni, A. Z. Incidence of myofascial pain syndrome in breast cancer surgery: a prospective study. Clin J Pain. 26, 320-325 https://doi.org/10.1097/AJP.0b013e3181c4904a (2010).

49. McPartland, J. M. Travell trigger points-molecular and osteopathic perspectives. J Am Osteopath Assoc. 104, 244-249 (2004).

50. Shah, J. P. \& Gilliams, E. A. Uncovering the biochemical milieu of myofascial trigger points using in vivo microdialysis: an application of muscle pain concepts to myofascial pain syndrome. J Bodyw Mov Ther. 12, 371-384 https://doi.org/10.1016/j.jbmt.2008.06.006 (2008).

51. Hsieh, R. L. \& Lee, W. C. Are the effects of botulinum toxin injection on myofascial trigger points placebo effects or needling effects? Arch Phys Med Rehabil. 89, 792-793 author reply 793 https://doi.org/10.1016/j.apmr.2008.02.004 (2008).

52. Tepper, S. J. Treatment of headache pain with botulinum neurotoxins. Pain Pract. 4 (Suppl 1), S3846 https://doi.org/10.1111/j.1533-2500.2004.04013.x (2004).

53. Ondo, W. G., Vuong, K. D. \& Derman, H. S. Botulinum toxin A for chronic daily headache: a randomized, placebo-controlled, parallel design study. Cephalalgia. 24, 60-65 https://doi.org/10.1111/j.1468-2982.2004.00641.x (2004).

54. Troost, B. T. Botulinum toxin type A (Botox) in the treatment of migraine and other headaches. Expert Rev Neurother. 4, 27-31 https://doi.org/10.1586/14737175.4.1.27 (2004).

55. Robertson, C. E. \& Garza, I. Critical analysis of the use of onabotulinumtoxinA (botulinum toxin type A) in migraine. Neuropsychiatr Dis Treat. 8, 35-48 https://doi.org/10.2147/NDT.S17923 (2012).

56. Ramachandran, R. \& Yaksh, T. L. Therapeutic use of botulinum toxin in migraine: mechanisms of action. Br J Pharmacol. 171, 4177-4192 https://doi.org/10.1111/bph.12763 (2014).

57. Steingrimsdottir, O. A., Kopke Vollestad, N. \& Knardahl, S. A prospective study of the relationship between musculoskeletal or psychological complaints and muscular responses to standardized cognitive and motor tasks in a working population. Eur J Pain. 9, 311-324 https://doi.org/10.1016/j.ejpain.2004.08.001 (2005).

58. Ustun, N. et al. Efficacy of EMLA cream phonophoresis comparison with ultrasound therapy on myofascial pain syndrome of the trapezius: a single-blind, randomized clinical study. Rheumatol Int. 34, 453-457 https://doi.org/10.1007/s00296-013-2881-2 (2014). 
59. Barbero, M. et al. Myofascial trigger points and innervation zone locations in upper trapezius muscles. BMC Musculoskelet Disord. 14, 179 https://doi.org/10.1186/1471-2474-14-179 (2013).

60. Sarrafzadeh, J., Ahmadi, A. \& Yassin, M. The effects of pressure release, phonophoresis of hydrocortisone, and ultrasound on upper trapezius latent myofascial trigger point. Arch Phys Med Rehabil. 93, 72-77 https://doi.org/10.1016/j.apmr.2011.08.001 (2012).

61. Vargas-Schaffer, G., Nowakowsky, M., Eghtesadi, M. \& Cogan, J. Ultrasound-Guided Trigger Point Injection for Serratus Anterior Muscle Pain Syndrome: Description of Technique and Case Series. A A Case Rep. 5, 99-102 https://doi.org/10.1213/XAA.0000000000000196 (2015).

62. De Groef, A. et al. Identification of Myofascial Trigger Points in Breast Cancer Survivors with Upper Limb Pain: Interrater Reliability. Pain Med. 19, 1650-1656 https://doi.org/10.1093/pm/pnx299 (2018).

63. Stubblefield, M. D. \& Keole, N. Upper body pain and functional disorders in patients with breast cancer. PM R. 6, 170-183 https://doi.org/10.1016/j.pmrj.2013.08.605 (2014).

64. De Groef, A. et al. Effectiveness of postoperative physical therapy for upper-limb impairments after breast cancer treatment: a systematic review. Arch Phys Med Rehabil. 96, 1140-1153 https://doi.org/10.1016/j.apmr.2015.01.006 (2015).

65. Gobel, H., Heinze, A., Heinze-Kuhn, K. \& Jost, W. H. Evidence-based medicine: botulinum toxin A in migraine and tension-type headache. J Neurol. 248 (Suppl 1), 34-38 https://doi.org/10.1007/pl00007818 (2001).

66. Dodick, D. W. Botulinum neurotoxin for the treatment of migraine and other primary headache disorders: from bench to bedside. Headache. 43 Suppl (1), S25-33 https://doi.org/10.1046/j.15264610.43.7s.5.x (2003).

67. Chan, V. W., McCabe, E. J. \& MacGregor, D. L. Botox treatment for migraine and chronic daily headache in adolescents. J Neurosci Nurs. 41, 235-243 https://doi.org/10.1097/jnn.0b013e3181aaa98f (2009).

68. Yi, K. H. et al. Intramuscular Neural Distribution of Rhomboid Muscles: Evaluation for Botulinum Toxin Injection Using Modified Sihler's Method. Toxins (Basel). 12, https://doi.org/10.3390/toxins12050289 (2020).

69. Wiater, J. M. \& Flatow, E. L. Long thoracic nerve injury. Clin Orthop Relat Res,17-27(1999).

70. Helgadottir, H., Kristjansson, E., Einarsson, E., Karduna, A. \& Jonsson, H. Jr. Altered activity of the serratus anterior during unilateral arm elevation in patients with cervical disorders. J Electromyogr Kinesiol. 21, 947-953 https://doi.org/10.1016/j.jelekin.2011.07.007 (2011).

71. Liem, R. S. \& van Douwe, J. In toto staining and preservation of peripheral nervous tissue. Stain Technol. 63, 113-120 https://doi.org/10.3109/10520298809107170 (1988).

\section{Figures}




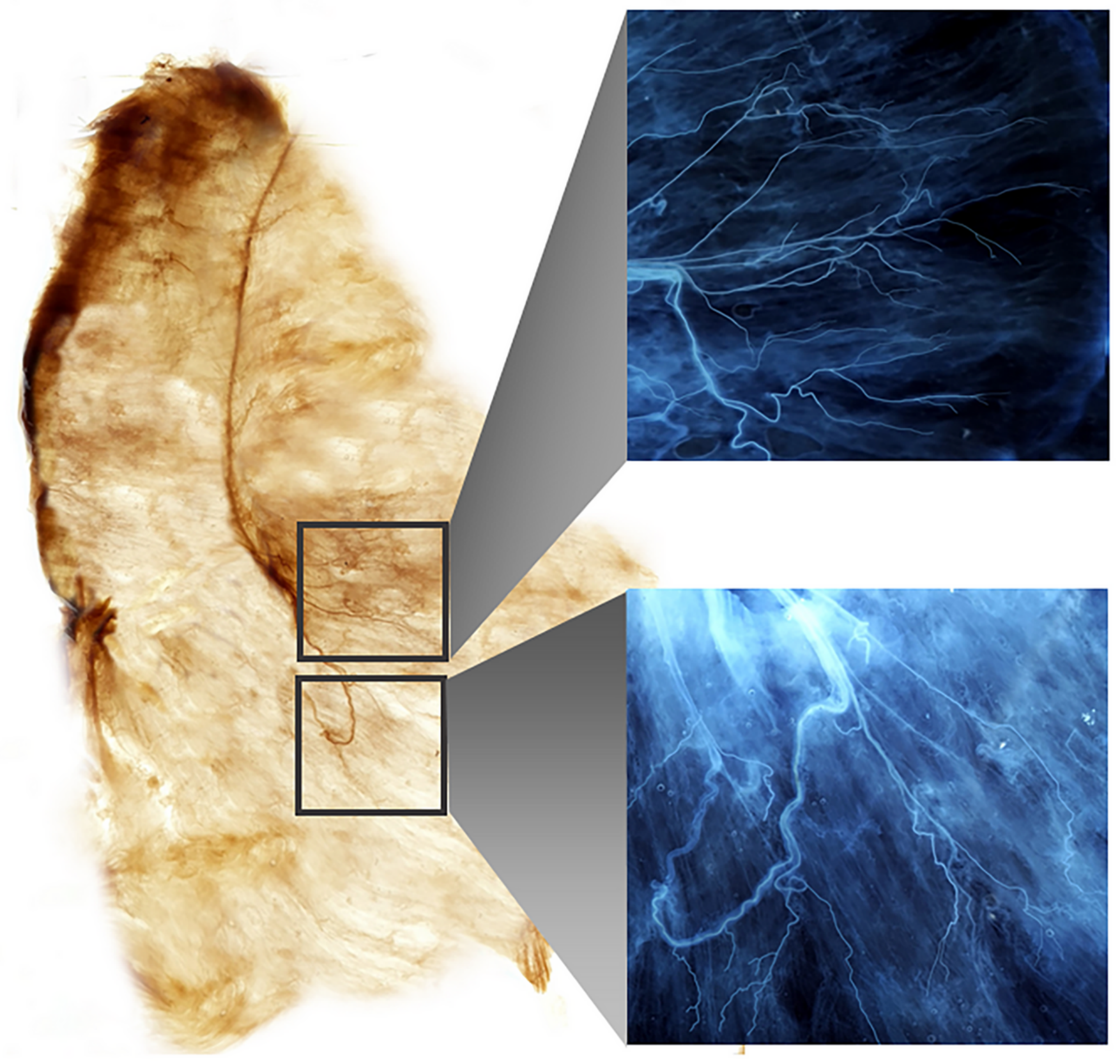

Figure 1

The result of Sihler's staining of the serratus anterior muscle. The intramuscular neural distribution of the serratus anterior muscle is observed with enlarged views. 


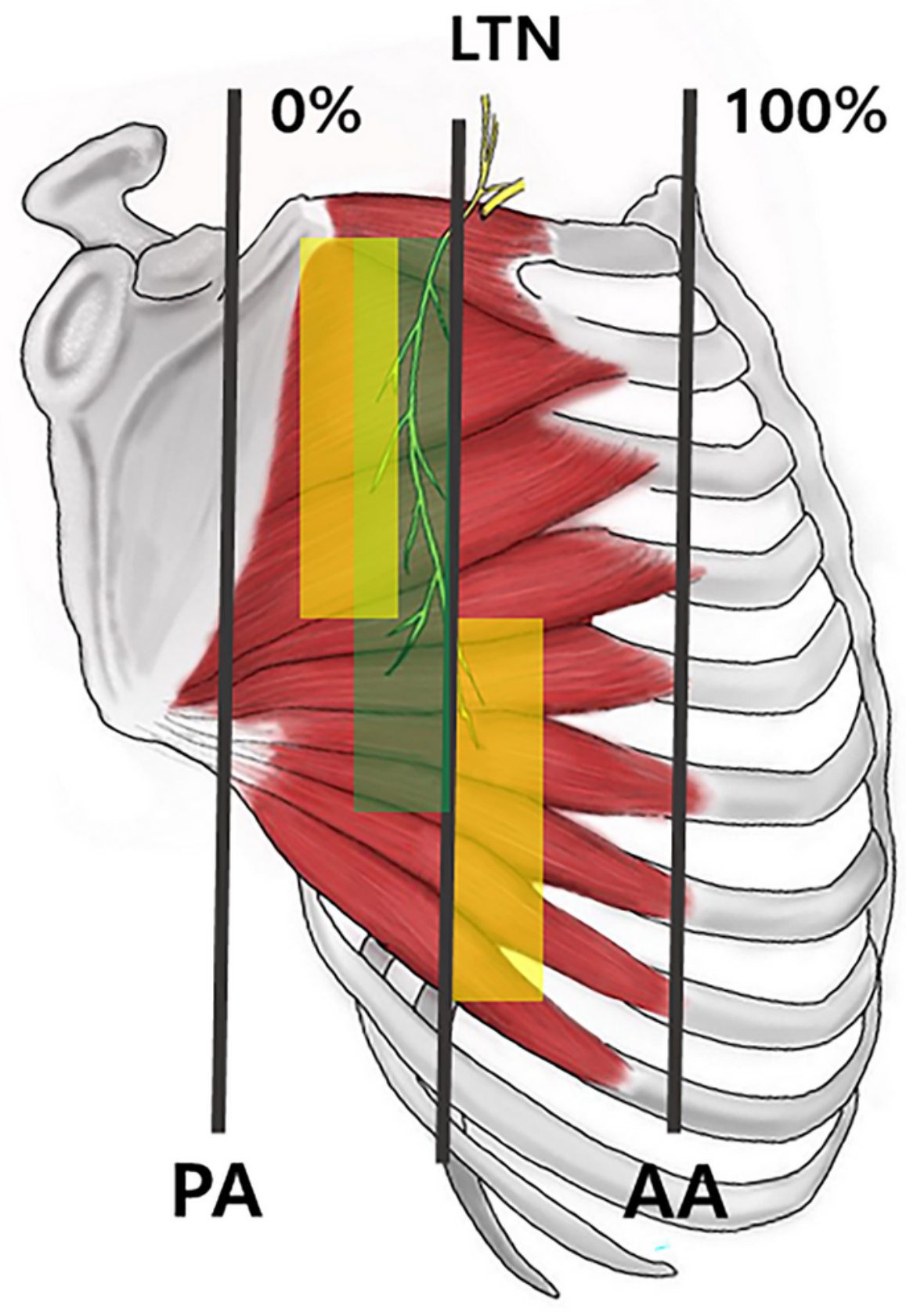

Figure 2

The long thoracic nerve runs superficial to the SA muscle and pierces into the muscle at each level until the 7th rib. The specimens had a trunk of the long thoracic nerve running on 30 to $40 \%$ throughout the level of 1st to 7th rib (green shaded). The intramuscular arborization patterns were the largest: in the 6th to 9 th rib portion had between $50 \%$ to $70 \%$ and the 1 st to 5 th rib portion had between 20 to $40 \%$. The injection should be guided to these arborized areas. 


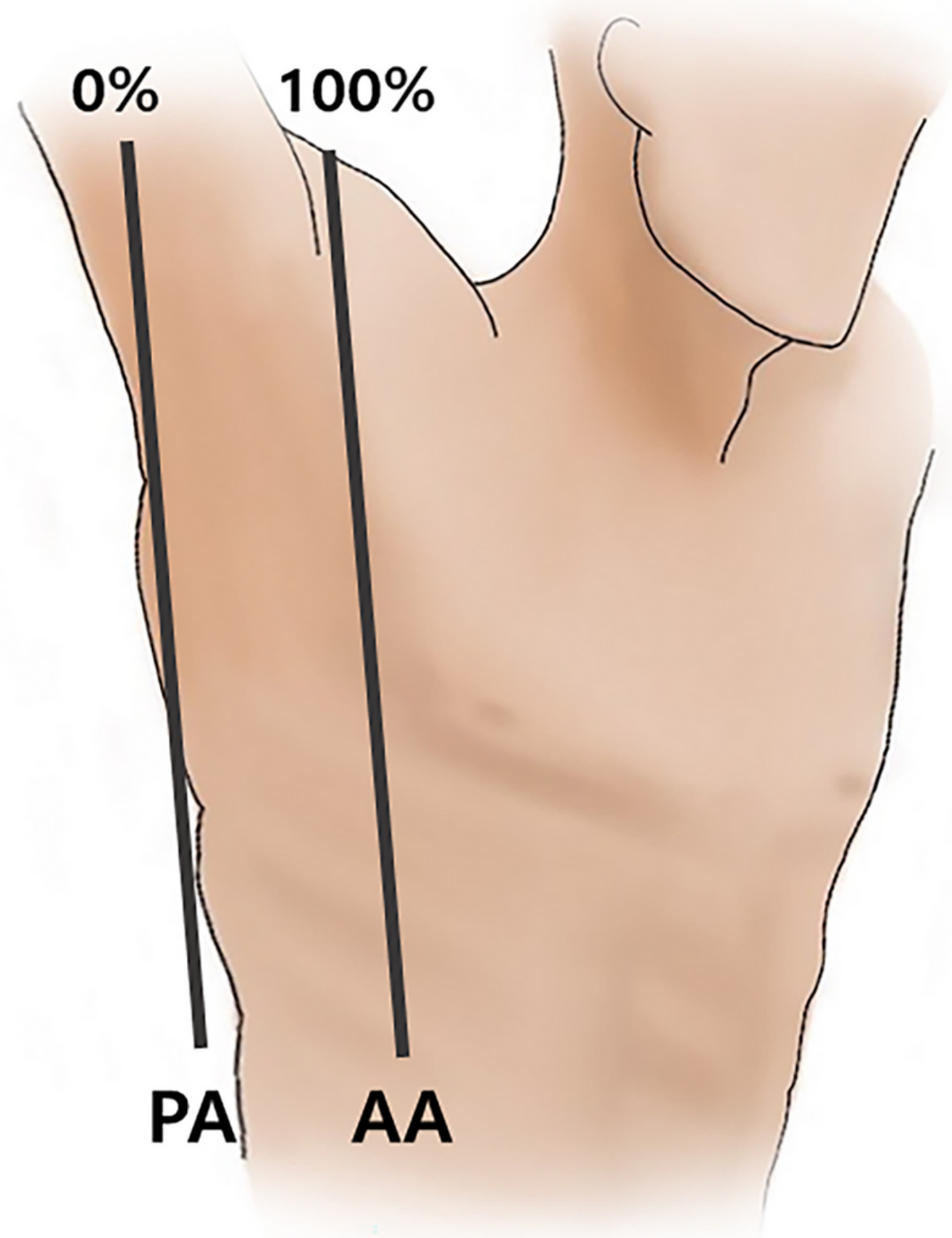

Figure 3

Specimens were harvested according to two anatomical lines: anterior axillary line (AA) and posterior axillary line (PA). The AA was $0 \%$ to PA of $100 \%$, respectively. 


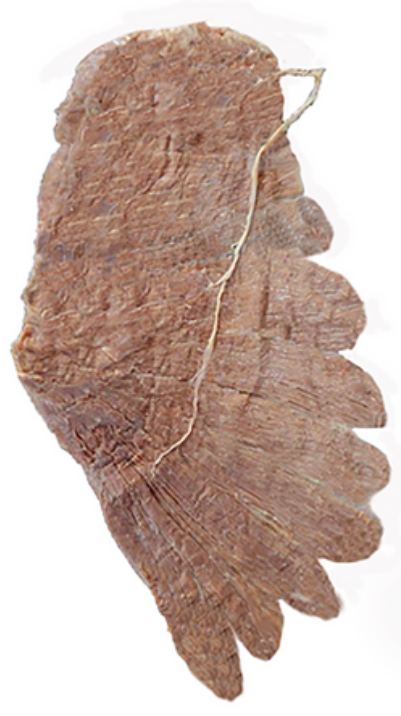

FX

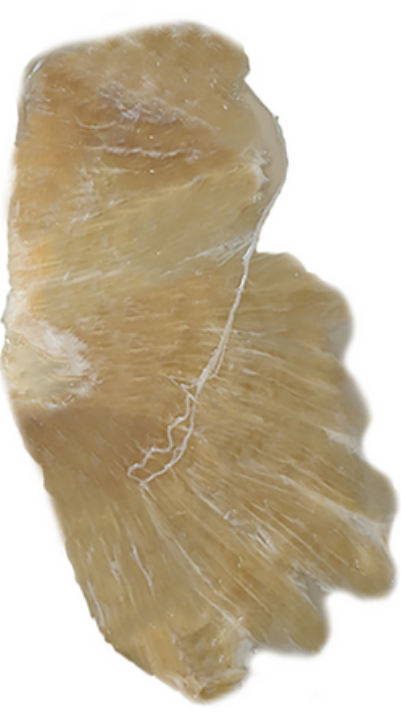

MD

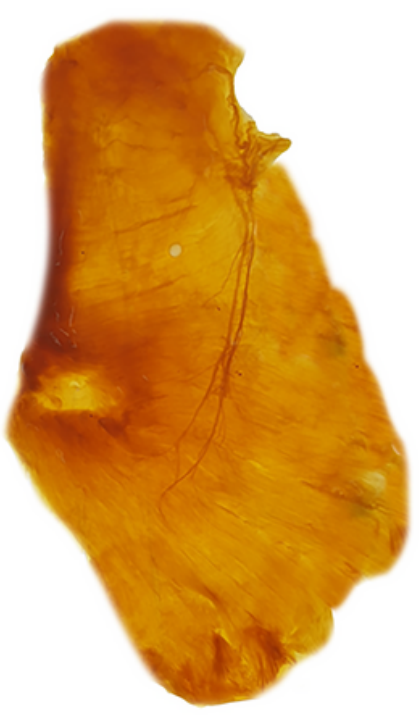

ST

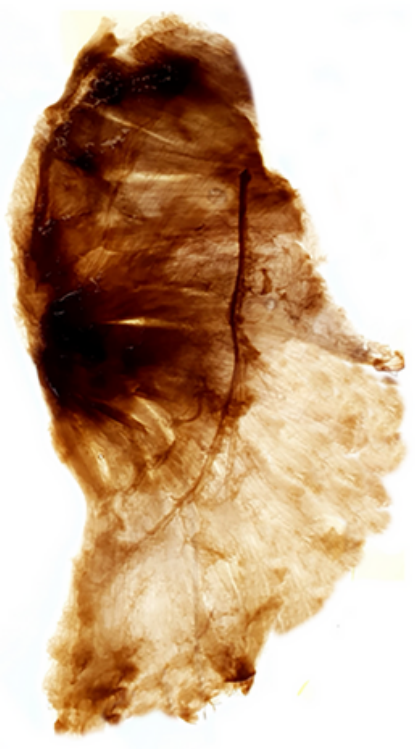

$\mathrm{CL}$

Figure 4

The serratus anterior muscle underwent modified Sihler's method. The method consists of stages of fixation (FX), maceration and depigmentation (MD), decalcification, staining (ST), and clearing (CL). 